\title{
Chaotic switching for quantum coin Parrondo's games with application to encryption
}

\author{
Joel Weijia Lai $\odot$ \\ Science, Mathematics and Technology, Singapore University of Technology and Design, 8 Somapah Road, Singapore 487372, Singapore \\ Kang Hao Cheong $\oplus^{*}$ \\ Science, Mathematics and Technology, Singapore University of Technology and Design, 8 Somapah Road, Singapore 487372, Singapore \\ and SUTD-MIT International Design Centre, 8 Somapah Road, Singapore 487372, Singapore
}

(Received 24 February 2021; accepted 18 April 2021; published 2 June 2021)

\begin{abstract}
Quantum game theory has stimulated some interest in recent years with the advancement of quantum information theory. This interest has led to a resurgence of quantum Parrondo's games. With two losing games combining to give a winning game, this paradoxical idea is known as Parrondo's paradox. By using chaotic switching between the two losing quantum games, we show that it is possible to achieve Parrondo's paradox involving a quantum walker playing two-sided quantum coin tossing games. Furthermore, we show that the framework of chaotic switching in quantum coin tosses can be applied to encryption. This is a proposal to deploy a quantum coin toss with chaotic switching for semiclassical encryption.
\end{abstract}

DOI: 10.1103/PhysRevResearch.3.L022019

Introduction. Parrondo's paradox refers to the phenomenon where two individually losing games can be combined to win through deterministic or random mixing of the games [1-7]. Since the introduction of the original capital-dependent Parrondo's games, there have been variants such as historydependent games [8] and cooperative games [9-11]. In recent years, quantum game theory has been gaining popular traction, especially quantum Parrondo's games [12-16]. Previous work showed chaotic switching to be superior for various optimization problems, with seminal papers that considered chaotic switching in classical Parrondo's games [17-21]. Classical Parrondo's games with chaotic switching and the potential to use controllable quantum systems to investigate certain fundamental aspects of nature [22-24] have motivated our study of applying chaotic switching to quantum Parrondo's games. Implementation of chaotic switching to quantum Parrondo's games critically narrows the gap in the literature to achieve a fullyimplementable quantum chaotic Parrondo's game.

Furthermore, this work seeks to amalgamate chaotic switching with quantum Parrondo's games. Chaotic switching, unlike random switching performed in previous research, is a reversible process without having to generate a register. Additionally, chaotic switching, unlike sequential switching performed in previous research, produces a result that is sensitive to initial conditions. Thus, while the chaotic sequence is deterministic, there is variability in producing such a se-

\footnotetext{
*kanghao_cheong@sutd.edu.sg

Published by the American Physical Society under the terms of the Creative Commons Attribution 4.0 International license. Further distribution of this work must maintain attribution to the author(s) and the published article's title, journal citation, and DOI.
}

quence by changing the initial condition. This places chaotic switching at an added advantage over previously studied random and sequential switchings, opening up avenues for applications to modern problems. We present one such application to encryption as an illustration of our work here.

The two-sided quantum coin toss random walk [15] will be the starting point of the quantum Parrondo's games to be investigated. The development of quantum random walk games ties in with the fundamental development of quantum computing; hence, research into quantum random walks continues to be an area of immense interest and motivates experimental implementations as well as theoretical advancement. Furthermore, a Parrondo paradoxical two-sided quantum coin toss random walk, while nontrivial, can be realized through modern standards of quantum computing [25].

In this paper, we show that chaotic switching can, indeed, improve on quantum coin tossing Parrondo's games and also provide insight into how chaotic switching for quantum coin tosses can be applied to encryption. We discuss the method behind developing a quantum coin toss Parrondo's game with chaotic switching. We show that Parrondo's paradox appears under chaotic switching. Finally, we show the use of such a protocol for application in encryption.

The Hilbert space in which the two-sided quantum coin toss random walk operates is $\mathcal{H}_{2} \otimes \mathcal{H}_{p}$, where $\mathcal{H}_{2}$ is the twosided coin space and $\mathcal{H}_{p}$ is the position space. The dynamics in the position space is coupled to the one in the coin space; however, the converse is not true. Each step of a quantum random walk comprises two transformations. The two transformations are (1) the flipping of a quantum coin (the coin operator $\hat{\mathcal{C}}$ ) and (2) the boost corresponding to the result of the first transformation (the translation operator $\hat{\mathcal{S}}$ ). A quantum coin is tossed to decide the number of discrete steps $s_{n}$ to take with reference to the result of the quantum coin flip. A general two-sided quantum coin is an arbitrary superposition of two 
states,

$$
|c\rangle=a_{0}|0\rangle_{c}+a_{1}|1\rangle_{c},\left|a_{0}\right|^{2}+\left|a_{1}\right|^{2}=1,
$$

with the basis

$$
|0\rangle_{c}=\left[\begin{array}{l}
1 \\
0
\end{array}\right], \quad|1\rangle_{c}=\left[\begin{array}{l}
0 \\
1
\end{array}\right]
$$

The transformation given by the coin operator $\hat{\mathcal{C}}$ takes the form of a $2 \times 2$ general unitary operator,

$$
\hat{C}(\rho, \alpha, \beta)=\left(\begin{array}{cc}
\sqrt{\rho} & \sqrt{1-\rho} e^{i \alpha} \\
\sqrt{1-\rho} e^{i \beta} & -\sqrt{\rho} e^{i \alpha \beta}
\end{array}\right),
$$

where $0 \leqslant \rho \leqslant 1,0 \leqslant \alpha, \beta \leqslant \pi$. The position of the walker on the line is represented as a superposition of the $p$ possible states,

$$
|x\rangle=\sum_{k} \alpha_{k}|k\rangle_{p}, \quad \sum_{k}\left|\alpha_{k}\right|^{2}=1,
$$

with the translation operator $\hat{\mathcal{S}}$ taking the following form:

$$
\hat{\mathcal{S}}=|0\rangle_{c}\left\langle 0\left|\otimes \sum_{k}\right| k+s_{0}\right\rangle_{p}\langle k|+| 1\rangle_{c}\left\langle 1\left|\otimes \sum_{k}\right| k+s_{1}\right\rangle_{p}\langle k|,
$$

where $s_{j}, j \in\{0,1\}$ is the number of steps taken by the walker in a random walk. Thus, the entire transformation is unitary and combines the coin operator and translation operator, given by

$$
\hat{\mathcal{U}}=\hat{\mathcal{S}}\left(\hat{\mathcal{C}} \otimes \hat{\mathcal{I}}_{p}\right),
$$

where $\hat{\mathcal{I}}_{p}$ is the identity operator of size $p \times p$. The initial state of the system is $|\psi\rangle_{0}=|c\rangle_{0} \otimes|x\rangle_{0}$, and $m$ steps are taken by applying the unitary operator $m$ times,

$$
|\psi\rangle_{m}=\hat{\mathcal{U}}^{m}|\psi\rangle_{0}
$$

The choice of operators and initial state is important in revealing the Parrondo effect. For this work, we choose the coin operators from Eq. (3) to take the form of the following Hadamard-like matrices:

$$
\hat{\mathcal{C}}_{A}=\frac{1}{\sqrt{2}}\left[\begin{array}{cc}
1 & 1 \\
1 & -1
\end{array}\right], \quad \hat{\mathcal{C}}_{B}=\frac{1}{\sqrt{2}}\left[\begin{array}{cc}
-1 & i \\
-i & 1
\end{array}\right] .
$$

In order to investigate the effects of chaotic switching, we set a known translation operator which has the form of Eq. (4), for which Parrondo's paradox is observed, specifically,

$$
\hat{\mathcal{S}}=|0\rangle_{c}\left\langle 0\left|\otimes \sum_{k}\right| k+1\right\rangle_{p}\langle k|+| 1\rangle_{c}\left\langle 1\left|\otimes \sum_{k}\right| k-1\right\rangle_{p}\langle k| .
$$

Note that while the chaotic switching determined by the maps generates the choice of quantum coin that is used, it is independent of the steps taken by the quantum random walker.

Finally, an initial state is chosen (not unique) for Parrondo's effect to emerge. We examine the effects of game $\mathrm{A}$ and game $\mathrm{B}$ under different switching schemes. The initial state considered is

$$
\begin{aligned}
|\psi\rangle_{0}= & -\frac{1}{2}\left[|0\rangle_{c} \otimes|2\rangle_{p}\right]+\frac{i}{2}\left[|1\rangle_{c} \otimes|0\rangle_{p}\right] \\
& +\frac{i}{2}\left[|0\rangle_{c} \otimes|0\rangle_{p}\right]-\frac{1}{2}\left[|1\rangle_{c} \otimes|-2\rangle_{p}\right] .
\end{aligned}
$$

Previous results from the two-sided quantum coin toss random walk Parrondo's games have shown that the random switching of two fair quantum coins, each with successive negative biased expectations, gives an average random walk position equivalent to the classical fair coin toss game [15]. Furthermore, the sequential periodic switching of two quantum coins, each with successive negative biased expectations, gives an average random walk with positive expectation.

Chaos is aperiodic long-term behavior in a deterministic system that exhibits sensitive dependence on initial conditions. While the irregularities of chaotic and random sequences in the time domain are often quite similar, they can be differentiated by their phase diagram. Consecutive points of a chaotic sequence are highly correlated (hence deterministic) but not in the case of a purely random sequence. A chaotic sequence, $X=\left\{x_{i}\right\}_{i=0}^{n}$, is generated by a map through iterative composition of the function $f_{n}(\cdot)$, that is,

$$
x_{n}=f_{n}\left(f_{n-1}\left(\cdots f_{2}\left(f_{1}\left(x_{0}\right)\right) \cdots\right)\right) .
$$

In this paper, we consider various chaotic maps as the means to generate chaotic switching. They are (1) a logistic map,

$$
x_{n+1}=a x_{n}\left(1-x_{n}\right),
$$

(2) a sinusoidal map,

$$
x_{n+1}=a x_{n}^{2} \sin \left(\pi x_{n}\right)
$$

and (3) a tent map,

$$
x_{n+1}= \begin{cases}a x_{n} & \text { if } x_{n} \leqslant 0.5, \\ a\left(1-x_{n}\right) & \text { otherwise. }\end{cases}
$$

Note that the logistic map and the tent map are topologically conjugate, as shown in Refs. [26,27]. Specifically, some fundamental aspects of chaotic dynamics are preserved under topological conjugacy. Effectively, showing that these chaotic maps are topologically conjugate allows us to collect different maps of equivalent classes and therefore facilitates the understanding of more complicated dynamical systems in terms of simpler ones, thus further expanding the applications of chaotic switching in quantum Parrondo's games. However, we will report our findings of all three maps for completeness.

Results. In the quantum coin random walk Parrondo's games, game $\mathrm{A}$ and game $\mathrm{B}$ are defined as the discrete-time tossing of quantum coins $\hat{\mathcal{C}}_{A}$ and $\hat{\mathcal{C}}_{B}$, respectively. At discretetime $n$, only one game will be played; that is, only quantum coin $\hat{\mathcal{C}}_{A}$ or $\hat{\mathcal{C}}_{B}$ will be used to determine the step taken by the quantum random walker. The choice of which coin to use follows an algorithm or pattern, which we call the switching strategy. We study the difference between the chaotic maps. By plotting the phase diagram and the bifurcation diagram of each of the chaotic maps (see Fig. 1), we are able to observe and choose the parameters to determine the switching dynamics for each map.

There are two main observations from Fig. 1. First, the bifurcation diagrams reveal how the control parameter $a$ determines the structure and order of switching. Take, for example, $a=3.2$ for the logistic map. Under such conditions, if we were to take the two branches of the bifurcation diagram, say, if $x_{n}<0.6$, we play game A; otherwise, we play game $\mathrm{B}$. Then we simply end up with the game sequence 


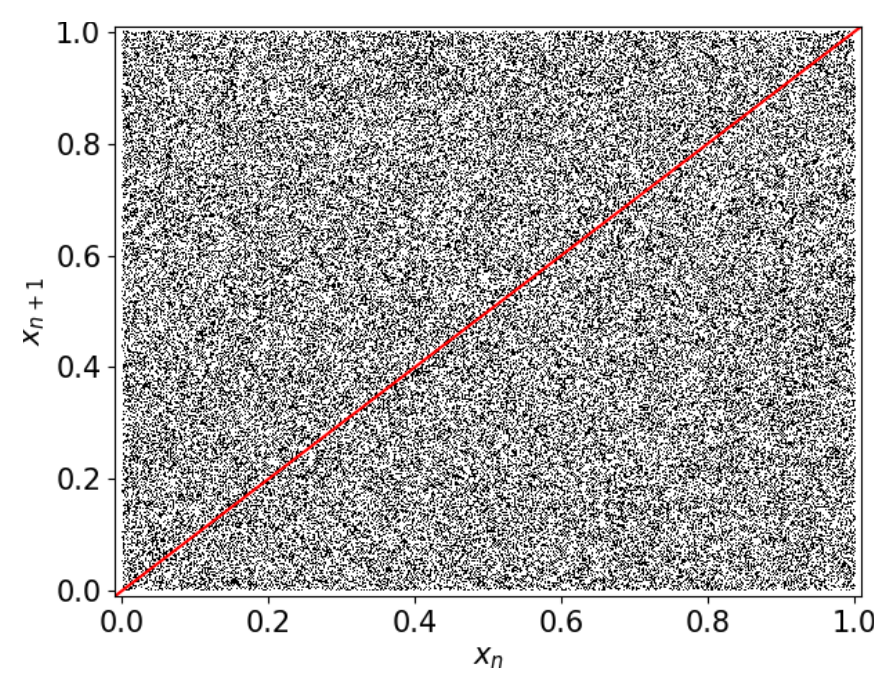

(a)
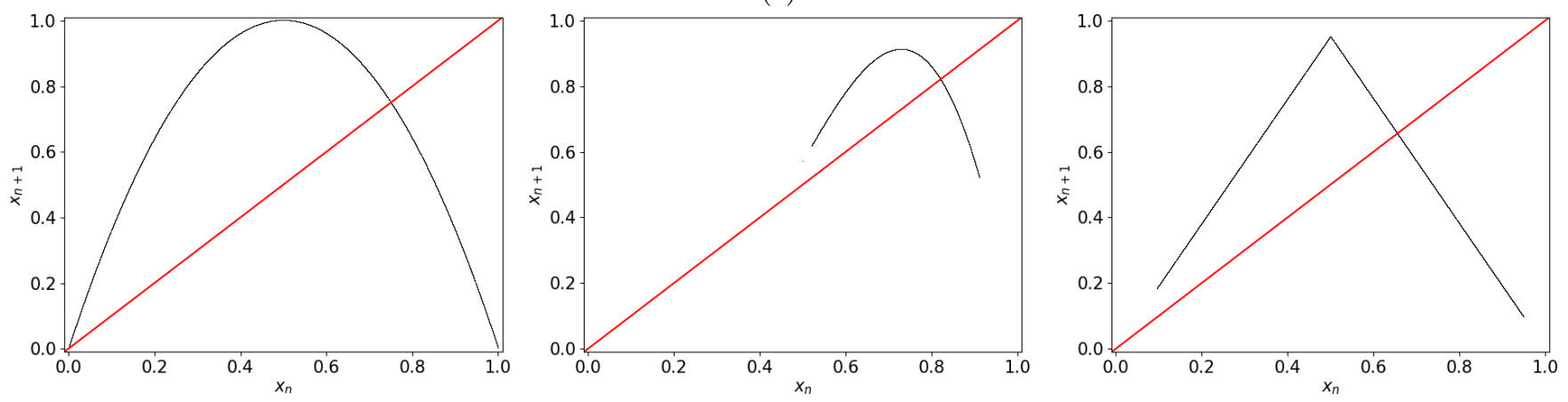

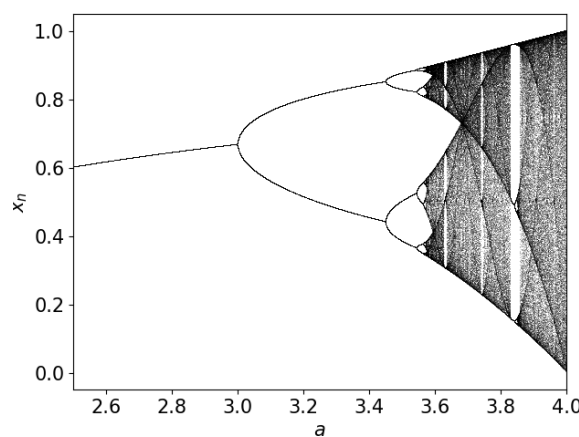

(b)

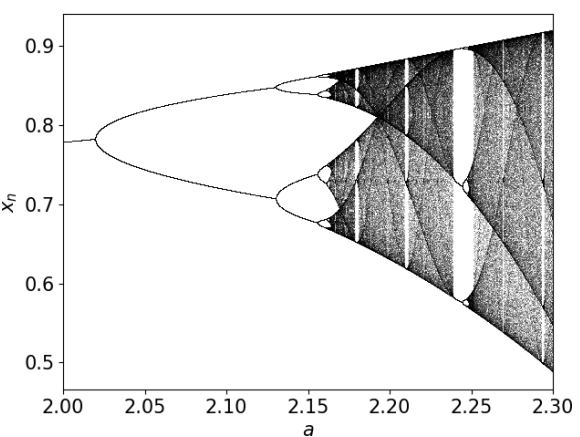

(c)

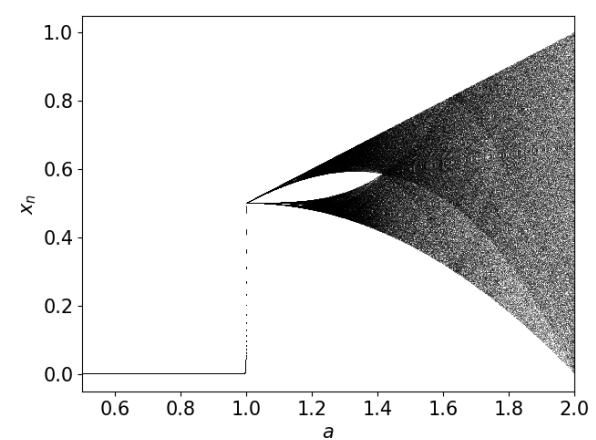

(d)

FIG. 1. (a) Phase diagram of a random map. Phase and bifurcation diagrams of the (b) logistic, (c) sinusoidal, and (d) tent maps. For the phase diagrams, the line $x_{n+1}=x_{n}$ is drawn to show the line of fixed points. The parameters chosen to generate the phase diagrams are $a=4$, $a=2.28$, and $a=1.9$, respectively, with the same initial condition used for the bifurcation diagrams, $x_{0}=0.1, x_{0}=0.5$, and $x_{0}=0.8$. The diagrams are generated by plotting the next 1000 values of $x_{n}$ after 100000 iterations.

$[\cdots$ AAAB $\cdots]$ as the transient periodic sequence, regardless of initial condition. However, if we take $a=4$, this sequence is still deterministic but is now sensitive to the initial condition (i.e., chaos). Second, in a comparison between the phase portrait of the random number generator and those of the chaotic maps, it is clear that despite being referred to as "chaotic" maps, there is structure and relation between $x_{n+1}$ and $x_{n}$. Thus, we can use "random map" (in a general sense) to refer to the case where $x_{n+1}$ and $x_{n}$ have no direct relation. The switching strategy will be determined by the chaotic sequence generated by the recurrence relation in Eqs. (12)-(14). The chaotic switching of game A and game B is investigated through simulations.

There are three parameters that are important in our discussion. The first parameter is $a$, which is the control parameter in each of the chaotic maps that determines whether the system is chaotic. For the purposes of investigation we identified the values of $a$ for which chaos occurs. The choices of $a$ are fixed; they are $a=4, a=2.28$, and $a=1.9$ for the logistic, sinusoidal, and tent maps, respectively. These values are chosen because by simple inspection of the bifurcation diagrams in Fig. 1 for these values of $a$, the maps are chaotic. The 


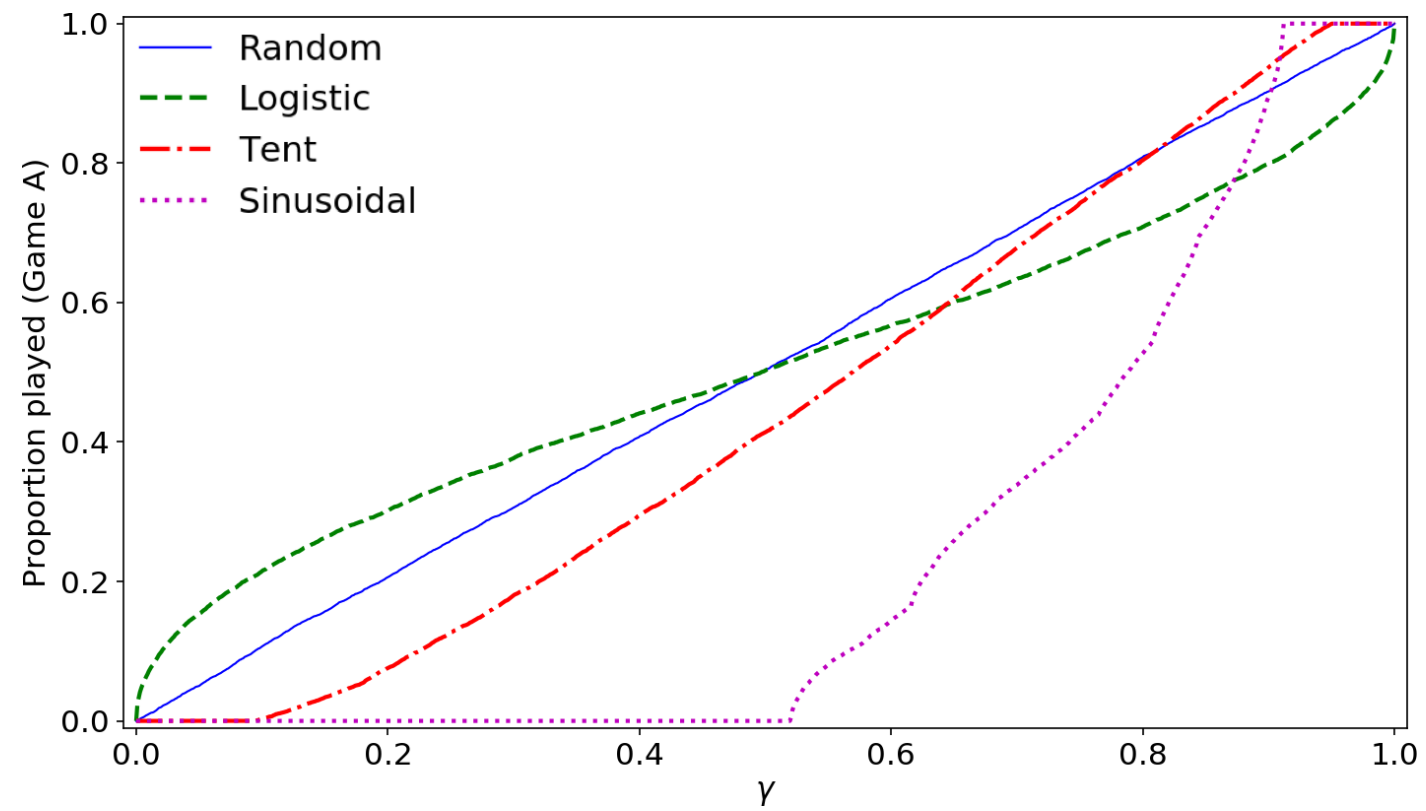

(a)

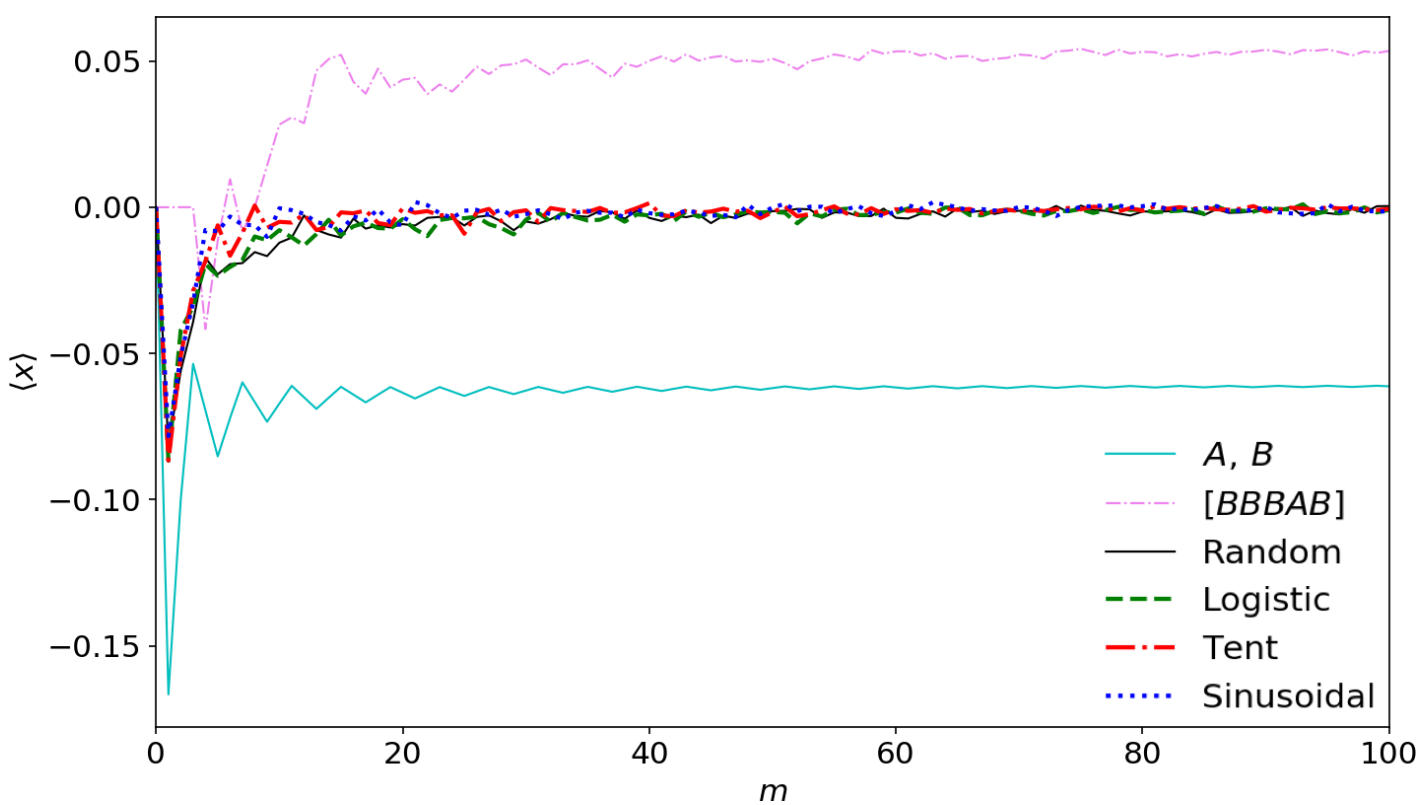

(b)

FIG. 2. (a) Plot of the effect parameter $\gamma$ on the proportion of game A played. As expected, the proportion of game A played against $\gamma$ for a uniform random switching is a straight line joining points $(0,0)$ and $(1,1)$. (b) The mean position of tossing quantum coins $\mathrm{A}$ and $\mathrm{B}$ individually, randomly, in a periodic sequence [BBBAB], and for each of the chaotic maps for $m=100$ steps.

second parameter is $x_{0}$, the initial value of $X$. Last, the third parameter is $\gamma$; this parameter controls the proportion of game A played. To determine whether to play game A or game B at each discrete time step $n$, we utilize the $\gamma$ parameter. The $\gamma$ value is important to make Parrondo's paradox appear. For example, in the case of the random map, $\gamma=0.5$ implies that game $\mathrm{A}$ and game $\mathrm{B}$ are played in equal proportion. In our model, if $x_{n}<\gamma$, then game $\mathrm{A}$ is played; otherwise, game B is played. As an example, consider $x_{0}=0.2$ and $\gamma=0.5$ for the logistic map with $a=4$. The sequence generated is $\{0.2,0.64,0.9216,0.289 \ldots, 0.821 \ldots, \ldots\}$, so the sequence of games played is $\{A, B, B, A, B, \ldots\}$. It is worth noting that by changing $x_{0}$ and $\gamma$, we are able to generate a different sequence, but all these sequences remain deterministic as they are generated by the logistic map with a fixed $a$. From this point forth, all sequences (i.e., steps taken) have length $m=100$.

This motivates the first simulation. We want to examine whether Parrondo's paradox appears in the case where games $\mathrm{A}$ and $\mathrm{B}$ are played with equal proportion for each of the chaotic maps. To determine the respective value of $\gamma$, we first plot a diagram to show the proportion of game A played.

From Fig. 2(a), we observe that the proportion of game A played is not linear in $\gamma$ for chaotic switching strategies. We 
TABLE I. Compilation of the mean positions and parameters used for both simulations.

\begin{tabular}{|c|c|c|c|c|}
\hline Map & $a$ & $x_{0}$ & $\gamma$ & $\langle x\rangle$ \\
\hline Game A/B & & & & -0.061261 \\
\hline $\begin{array}{l}\text { Random } \\
{[\mathrm{BBBAB}]}\end{array}$ & & $U(0,1)$ & 0.5 & $\begin{array}{c}3.9932 \times 10^{-4} \\
0.053479\end{array}$ \\
\hline \multicolumn{5}{|c|}{ Simulation 1} \\
\hline Logistic & 4 & 0.1 & 0.487 & $-8.8423 \times 10^{-4}$ \\
\hline Sinusoidal & 2.28 & 0.5 & 0.788 & -0.0013614 \\
\hline Tent & 1.9 & 0.8 & 0.569 & $-6.1492 \times 10^{-4}$ \\
\hline \multicolumn{5}{|c|}{ Simulation 2} \\
\hline Logistic & 4 & 0.5 & 0.6 & 0.090191 \\
\hline Sinusoidal & 2.28 & 0.96 & 0.24 & 0.023668 \\
\hline Tent & 1.9 & 0.98 & 0.08 & 0.044325 \\
\hline
\end{tabular}

found the value of $\gamma$ that results in an equal proportion of games $\mathrm{A}$ and $\mathrm{B}$ being played. The parameter values used in the first simulation are reported in Table I.

For all simulations, we have fixed the parameter $a$ for each of the chaotic maps. With the initial state from Eq. (10), our numerical experiments confirm that tossing quantum coin $\hat{\mathcal{C}}_{A}$ or coin $\hat{\mathcal{C}}_{B}$ individually results in a net negative mean position for the random walker after $m=100$ steps. Simulations were carried out for the random map and all periodic sequences of up to length 5; of importance is the sequence [BBBAB]. Subjected to the quantum coins $\hat{\mathcal{C}}$ and the initial condition $|\psi\rangle_{0}$, the periodic sequence [BBBAB] produces the best positive mean position $\langle x\rangle$. The random map and the periodic sequence [BBBAB] will be used as benchmarks to evaluate the performance of the chaotic maps.

To simulate the effect of the chaotic maps, we randomly selected a subsequence of length 100 from each of the chaotic maps and computationally calculated the mean position of the random walker subjected to this sequence of games; that is, game $\mathrm{A}$ is played when $x_{n}<\gamma$. This is averaged over 300 randomly selected subsequences. The results are presented in Fig. 2(b).

As expected, the mean position of the random walker under the chaotic maps produces an expected position similar to that of a random map (fair outcome). This is due to the construct of the simulation. The choice between tossing quantum coins $A$ and $B$ is determined by averaging over 300 randomly selected subsequences. This random selection and averaging of subsequences nullifies the deterministic outcome that can be predicted from a chaotic sequences for a fixed $x_{0}$, thus giving us the same result as simply randomly choosing between tossing two quantum coins. In a previous paper [15], we showed that based on the construction of both quantum coins, they are inherently fair. Randomness, in this case averaging over 300 randomly selected subsequences, eliminates the quantum bias that emerges as a result of quantum coin tosses. Furthermore, since the classical limit of random tossing must reveal the underlying fair nature of the quantum coins, all mean positions of the random walker under the chaotic maps produce a fair outcome (i.e., mean position of zero). In this case, we see that the periodic sequence produces the highest mean position for the random walker. At this juncture, it is important to ask if we can do better.

With the knowledge that averaging over randomly selected subsequences results in a fair outcome, we now conduct the second simulation, which eliminates the process by which averaging takes place. Instead, we will use deterministic sequences that are generated by each of the chaotic maps. To find the values of $x_{0}$ and $\gamma$ that produce the best possible mean position, we use the same initial state and values of $a$ as in the previous simulation and plot the mean position of the random walker after $m=100$ steps for various values of $x_{0}$ and $\gamma$ in the form of a heat map. As it turns out that we can actually do better, the results are shown in Fig. 3.

From Figs. 3(a) and 3(b), we can determine the values of $x_{0}$ and $\gamma$ for each chaotic map that return the best outcome for the random walker for their respective values of $a$. Using these parameter values (see parameter values in Table I), we perform a second simulation by applying the chaotic switching using the sequence generated by the values of $x_{0}$ and $\gamma$ in the initial state given by Eq. (10) using quantum coins, Eq. (8), and translation operator, Eq. (9). Similar to the procedure in simulation 1, if $x_{n}<\gamma$, quantum coin $\mathrm{A}$ is used; otherwise, coin B is used. The results are reported in Fig. 3(d).

The results from the second simulation show that the chaotic switching strategies now outperform that of the random switching strategy; in particular, the logistic map chaotic switching strategy outperforms the [BBBAB] periodic switching strategy. The numerical results of the first and second simulations are organized in Table I. The key difference between simulations 1 and 2 is the absence of averaging chaotic subsequences in the latter. Simulation 2 also varies $x_{0}$ and $\gamma$ to allow us to find the best possible combination of $x_{0}$ and $\gamma$ that gives the best outcome for the quantum random walker.

Conclusion. Developments in quantum Parrondo's games thus far seek to translate quantum games from their classical counterpart [14] or explore Parrondo's paradox with no classical analog. Previous research focused mainly on fundamental concepts in quantum information that may be useful in classifying operations or states in a quantum computer based on the final observed outcome. In previous work $[12,13,15,16]$, the quantum Parrondo's paradox emerged from a random sequence. However, since the sequence of coin tosses is random, one cannot extract information about the sequence without deploying significant computational memory. That is to say, if one were to ask what the final outcome from a particular start state would be or what sequence would result in a given final outcome, the answer to these questions could not be answered without performing discrete time computation and memory storage. However, in our framework and protocol (say logistic map), the sequence is generated by just two numbers, $x_{0}$ and $\gamma$. Therefore, given $x_{0}$ and $\gamma$, we will know the final outcome. Furthermore, we will also know the exact sequence to achieve the final outcome if we know $x_{0}$ or $\gamma$. Equivalently, if we know the final outcome and $\gamma$, we are able to use the exact inverse function iteratively to obtain $x_{0}$ without needing to know what the last toss was as there are only two sequences that need to be tested-when the final toss uses quantum coin A or B. This is the reason why using a random sequence is not feasible as there is no inverse to a random map. 


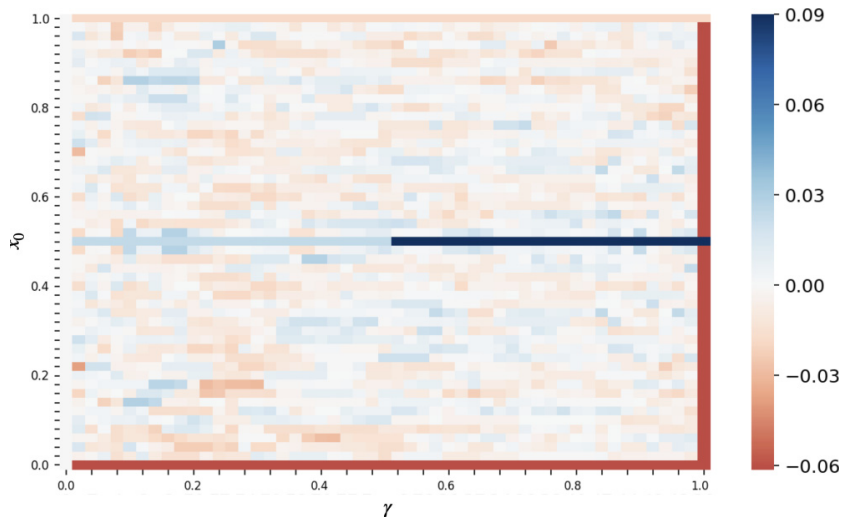

(a)

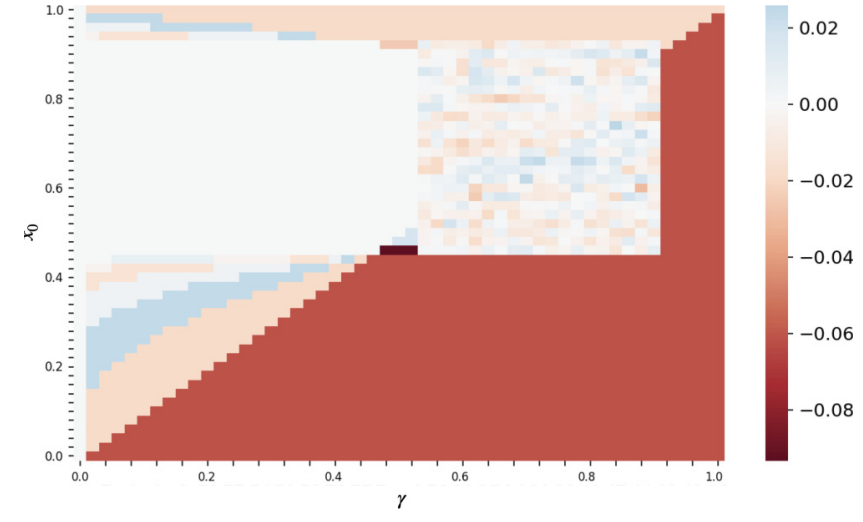

(b)

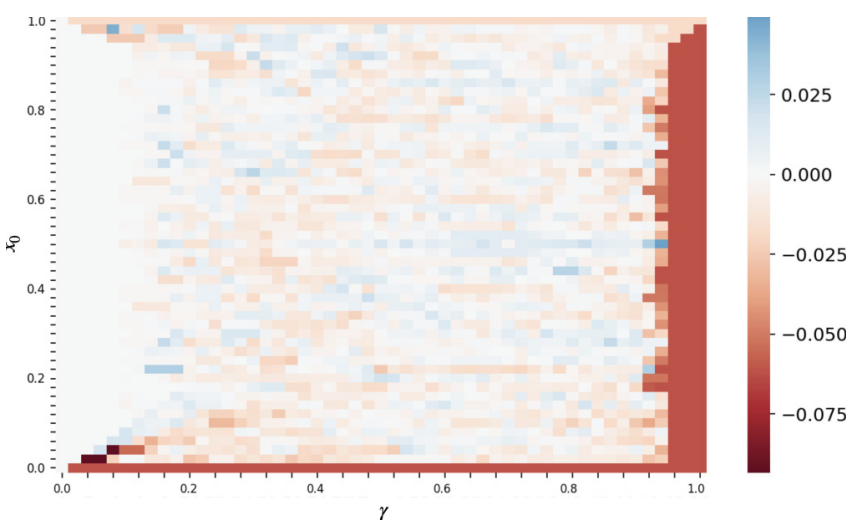

(c)

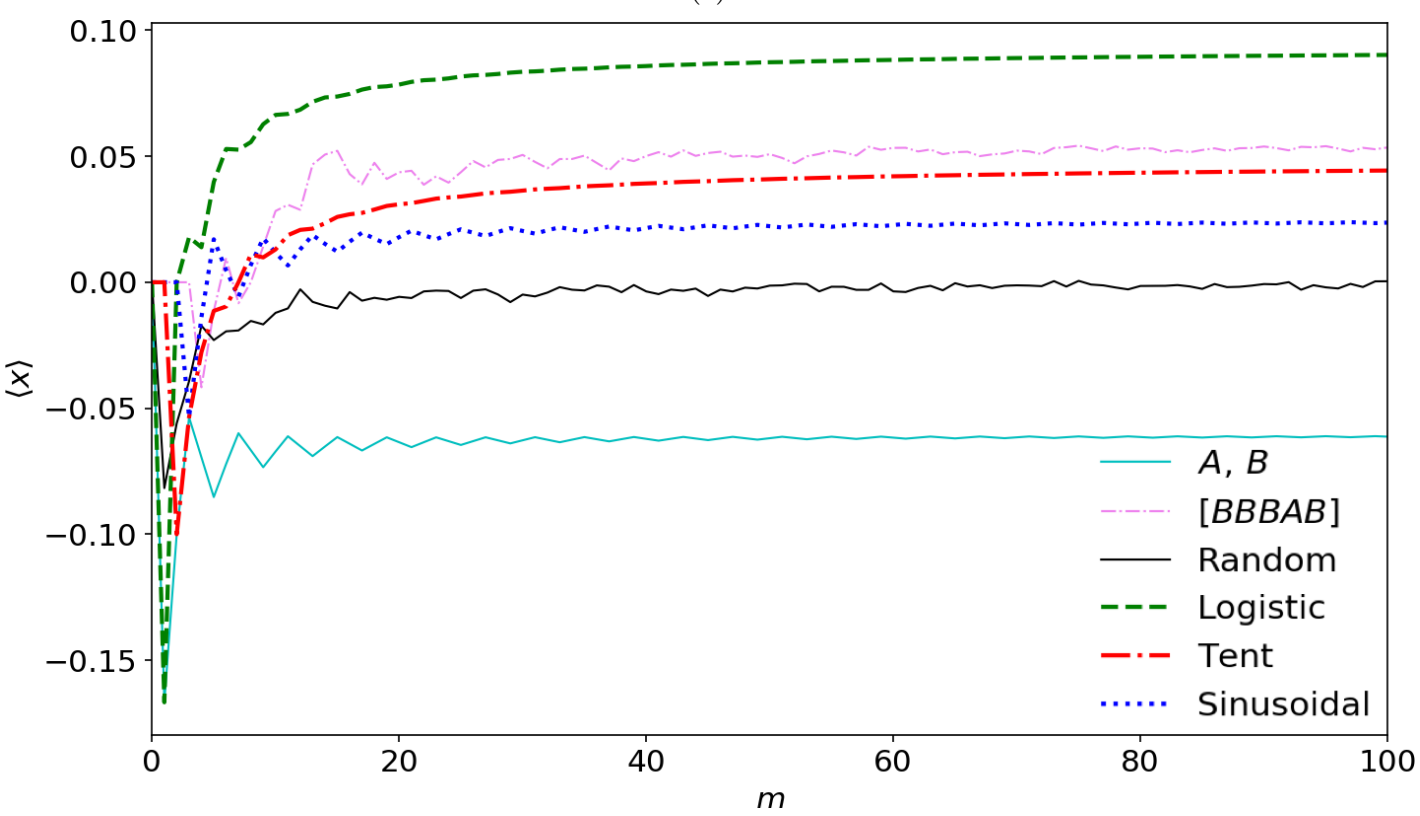

(d)

FIG. 3. Mean position of the random walker for each of the chaotic maps for $m=100$ steps by varying $x_{0}$ and $\gamma$. (a) Heat map of the logistic map for $a=4$. (b) Heat map of the sinusoidal map for $a=2.28$. (c) Heat map of the tent map for $a=1.9$. The values of $x_{0}$ and $\gamma$ vary in the closed interval $[0,1]$. (d) The mean position of the random walker from tossing quantum coins A and B individually, randomly, and in a periodic sequence [BBBAB] and the best outcome for the random walker for each chaotic map by varying the parameters $\gamma$ and $x_{0}$ in (a)-(c). 


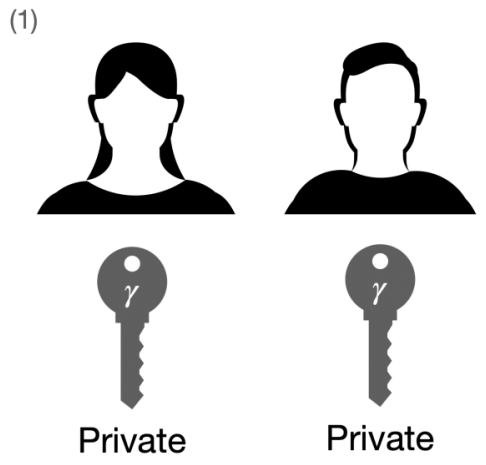

Alice and Bob both obtain a private key through a secure channel.

(2)

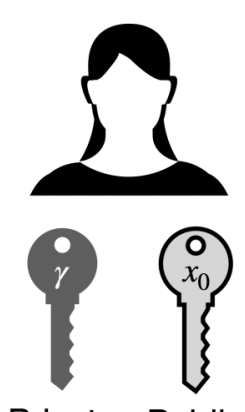

Private Public

The sender (Alice) generates a public key.
(3)

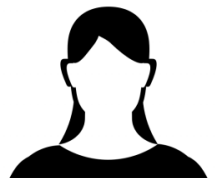

Alice obtains a chaotic sequence which is generated with $x_{0}$ and $\gamma$ from a pre-agreed chaotic map. The original message

$|\psi\rangle_{0}$ is scrambled using chaotic switching of quantum coins. The encrypted message is $|\psi\rangle_{f}$.

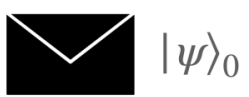

$|\psi\rangle_{f}$ and $x_{0}$ is then sent through an insecure channel to Bob.
(4)
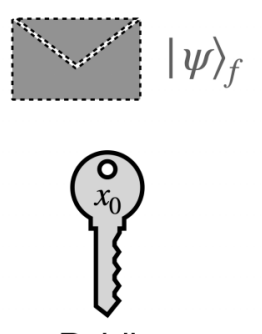

Public
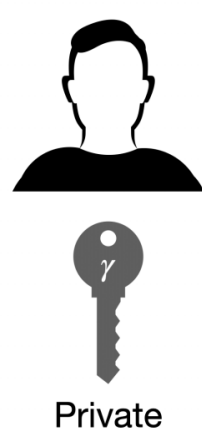

Since Bob has both keys, he is able to unscramble the message as chaotic sequences are deterministic. Bob deciphers the original message.

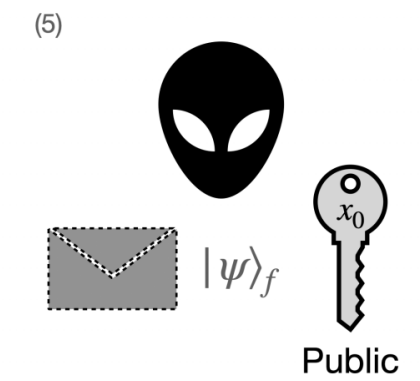

Interceptors are unable to decrypt the message as they require the private key.

FIG. 4. General schematic of how Parrondo's chaotic switching in quantum coin toss games can be applied to encryption through the use of secure and insecure channels.

This technique of determining which quantum coin to use by comparing $x_{n}$ to $\gamma$ can form the motivating basis for advances in encryption [28,29]. As an illustration of the use of such a protocol in encryption, consider a sender (Alice) and a receiver (Bob). The general schematic that makes use of Parrondo's chaotic switching in quantum coin toss games applied to encryption is summarized in Fig. 4.

Consider Alice and Bob, both of whom are able to receive a private key $\gamma$ through a secure channel. Here we assume that $\hat{\mathcal{C}}$ and $\hat{\mathcal{S}}$ are publicly known. Before Alice sends a message, she generates a public key $x_{0}$. With the generated $x_{0}$ and private $\gamma$, Alice is now able to generate a chaotic sequence. She uses this sequence, which determines a chaotic sequence of quantum coin tosses, to encrypt an initial state $|\psi\rangle_{0}$; this is the message that Alice would like to send. This encrypted message $|\psi\rangle_{f}$ and the public key $x_{0}$ are then sent through an insecure channel to Bob. The receiver, who holds the private key $\gamma$, will receive the final state $|\psi\rangle_{f}$ and public key $x_{0}$. The receiver is able to decipher the original message by using the received $x_{0}$ and $\gamma$ to reverse the final state to decipher the initial state, thus obtaining the original message. Such a scheme ensures that even if $|\psi\rangle_{f}$ and $x_{0}$ are intercepted and $\hat{\mathcal{C}}$ and $\hat{\mathcal{S}}$ are publicly known, the interceptor cannot efficiently decrypt them to obtain the original message. The complexity to decrypt (from the perspective of an interceptor) can be enhanced by including both $\hat{\mathcal{C}}$ and $\hat{\mathcal{S}}$ as private keys, but at the expense of having more preshared information. Notice that a similar effect can be achieved if, instead of using chaotic maps, a periodic switching is used. Thus, instead of the private key being $\gamma$, the private key is now the periodic sequence. In such a case, there is no requirement for a public key. However, this is a cipher that, once broken, cannot be reused. A disclosure of the periodic sequence renders all messages encrypted using this sequence unsecured.

In summary, the rate of winning obtained from a chaotic switching strategy is controlled by the parameters $a$ defining the chaotic generator, initial conditions $x_{0}$, and the proportion of game A played $\gamma$. We note that by varying the parameters $x_{0}$ and $\gamma$, Parrondo's games with chaotic switching strategy can give a higher rate of winning compared to a random switching strategy. This work brings us closer to finding the best possible mean position in a two-sided quantum coin random walk Parrondo's game. Additionally, while chaos in quantum systems is not defined in the same way as it is in chaotic and dynamical systems, there are quantum systems whose physical properties exhibit properties of chaos [30]. Furthermore, there is no concept of entanglement within classical systems. Yet research has shown that chaos and entanglement are very strongly and clearly related [22,23]. These results leapfrog the gap between the current status of quantum Parrondo's paradox and a fully implementable and demonstrable quantum-entangled Parrondo's paradox.

The introduction of chaotic switching, when combined with Parrondo's paradox, extends the application of Parrondo's paradox from simply a tool used in quantum information for classification or identification (of the initial 
state and final outcome) to one that has real-world engineering applications - as we can now also control the process of reaching the final outcome as observed in its proposed use in encryption. The development of a fully implementable quantum chaotic Parrondo's game may also improve on our semiclassical framework and provide advances to bridge some of the problems still faced in quantum encryption [31,32].
The wide application of quantum Parrondo's paradox puts our research in the spotlight, for this work also inspires future study of applications beyond the quantum Parrondo's paradox to explain physical phenomena.

Acknowledgment. This project was funded by the Singapore University of Technology and Design Grant No. SRG SCI 2019142.
[1] G. P. Harmer and D. Abbott, Losing strategies can win by Parrondo's paradox, Nature (London) 402, 864 (1999).

[2] K. H. Cheong, J. M. Koh, and M. C. Jones, Paradoxical survival: Examining the Parrondo effect across biology, BioEssays 41, 1900027 (2019).

[3] J. M. Koh and K. H. Cheong, Generalized solutions of Parrondo's games, Adv. Sci. 7, 2001126 (2020).

[4] K. H. Cheong, T. Wen, and J. W. Lai, Relieving cost of epidemic by Parrondo's paradox: A covid-19 case study, Adv. Sci. 7, 2002324 (2020).

[5] Z.-X. Tan, J. M. Koh, E. V. Koonin, and K. H. Cheong, Predator dormancy is a stable adaptive strategy due to Parrondo's paradox, Adv. Sci. 7, 1901559 (2020).

[6] K. H. Cheong, D. B. Saakian, and R. Zadourian, Allison mixture and the two-envelope problem, Phys. Rev. E 96, 062303 (2017).

[7] K. H. Cheong, Z. X. Tan, and Y. H. Ling, A time-based switching scheme for nomadic-colonial alternation under noisy conditions, Commun. Nonlinear Sci. Numer. Simul. 60, 107 (2018).

[8] J. M. R. Parrondo, G. P. Harmer, and D. Abbott, New Paradoxical Games Based on Brownian Ratchets, Phys. Rev. Lett. 85, 5226 (2000).

[9] R. Toral, Capital redistribution brings wealth by Parrondo's paradox, Fluct. Noise Lett. 2, L305 (2002).

[10] J. W. Lai and K. H. Cheong, Social dynamics and Parrondo's paradox: A narrative review, Nonlinear Dyn. 101, 1 (2020).

[11] J. W. Lai, J. Chang, L. Ang, and K. H. Cheong, Multi-level information fusion to alleviate network congestion, Inf. Fusion 63, 248 (2020).

[12] J. Rajendran and C. Benjamin, Implementing Parrondo's paradox with two-coin quantum walks, R. Soc. Open Sci. 5, 171599 (2018).

[13] J. Rajendran and C. Benjamin, Playing a true Parrondo's game with a three-state coin on a quantum walk, Europhys. Lett. 122, 40004 (2018).

[14] J. W. Lai and K. H. Cheong, Parrondo's paradox from classical to quantum: A review, Nonlinear Dyn. 100, 849 (2020).

[15] J. W. Lai and K. H. Cheong, Parrondo effect in quantum cointoss simulations, Phys. Rev. E 101, 052212 (2020).

[16] J. W. Lai, J. R. A. Tan, H. Lu, Z. R. Yap, and K. H. Cheong, Parrondo paradoxical walk using four-sided quantum coins, Phys. Rev. E 102, 012213 (2020).
[17] P. Arena, S. Fazzino, L. Fortuna, and P. Maniscalco, Game theory and non-linear dynamics: The Parrondo paradox case study, Chaos Soliton Fract. 17, 545 (2003).

[18] M. Bucolo, R. Caponetto, L. Fortuna, M. Frasca, and A. Rizzo, Does chaos work better than noise? IEEE Circ. Syst. Mag. 2, 4 (2002).

[19] T. W. Tang, A. Allison, and D. Abbott, Investigation of chaotic switching strategies in Parrondo's games, Fluct. Noise Lett. 4, L585 (2004).

[20] M.-F. Danca, M. Fečkan, and M. Romera, Generalized form of Parrondo's paradoxical game with applications to chaos control, Int. J. Bifurcation Chaos 24, 1450008 (2014).

[21] G. Paneru, D. Y. Lee, T. Tlusty, and H. K. Pak, Lossless Brownian Information Engine, Phys. Rev. Lett. 120, 020601 (2018).

[22] C. Neill et al., Ergodic dynamics and thermalization in an isolated quantum system, Nat. Phys. 12, 1037 (2016).

[23] C. J. Turner, A. A. Michailidis, D. A. Abanin, M. Serbyn, and Z. Papić, Weak ergodicity breaking from quantum many-body scars, Nat. Phys. 14, 745 (2018).

[24] S. Ito, Stochastic Thermodynamic Interpretation of Information Geometry, Phys. Rev. Lett. 121, 030605 (2018).

[25] M. Jan, Q.-Q. Wang, X.-Y. Xu, W.-W. Pan, Z. Chen, Y.-J. Han, C.-F. Li, G.-C. Guo, and D. Abbott, Experimental realization of Parrondo's paradox in 1D quantum walks, Adv. Quantum Technol. 3, 1900127 (2020).

[26] K. T. Alligood, T. D. Sauer, and J. A. Yorke, Chaos: An Introduction to Dynamical Systems (Springer, Berlin, 1997), pp. 114-124.

[27] R. Venegeroles, On the topological conjugacy problem for interval maps, arXiv:1411.3066.

[28] S. Tayal, N. Gupta, P. Gupta, D. Goyal, and M. Goyal, A review paper on network security and cryptography, Adv. Computa. Sci. Technol. 10, 763 (2017).

[29] N. Jirwan, A. Singh, and S. Vijay, Review and analysis of cryptography techniques, Int. J. Sci. Eng. Res. 4, 1 (2013).

[30] F. Carollo, F. M. Gambetta, K. Brandner, J. P. Garrahan, and I. Lesanovsky, Nonequilibrium Quantum Many-Body Rydberg Atom Engine, Phys. Rev. Lett. 124, 170602 (2020).

[31] S. Pirandola, U. L. Andersen, L. Banchi, M. Berta, D. Bunandar, R. Colbeck, D. Englund, T. Gehring, C. Lupo, C. Ottaviani, J. L. Pereira, M. Razavi, J. S. Shaari, M. Tomamichel, V. C. Usenko, G. Vallone, P. Villoresi, and P. Wallden, Advances in quantum cryptography, Adv. Opt. Photonics 12, 1012 (2020).

[32] V. Scarani and C. Kurtsiefer, The black paper of quantum cryptography: Real implementation problems, Theor. Comput. Sci. 560, 27 (2014). 